\title{
Real-time characterization of spectral coherence of ultrafast laser based on optical time-stretch
}

Yiqing Xu, Xiaoming Wei, Zhibo Ren, Kenneth K. Y. Wong, Kevin Tsia

Yiqing Xu, Xiaoming Wei, Zhibo Ren, Kenneth K. Y. Wong, Kevin Tsia, "Realtime characterization of spectral coherence of ultrafast laser based on optical time-stretch," Proc. SPIE 9732, Real-time Measurements, Rogue Events, and Emerging Applications, 97320C (9 March 2016); doi: 10.1117/12.2211969 


\title{
Real-time characterization of spectral coherence of ultrafast laser based on optical time-stretch
}

\author{
Yiqing Xua ${ }^{\mathrm{a}}$, Xiaoming Wei ${ }^{\mathrm{a}}$, Zhibo Ren ${ }^{\mathrm{a}}$, Kenneth K. Y. Wong ${ }^{\mathrm{a}}$, and Kevin Tsia ${ }^{\mathrm{a},}{ }^{*}$ \\ ${ }^{a}$ Department of Electrical and Electronic Engineering, The University of Hong Kong, Pokfulam \\ Road, Hong Kong, P. R. of China. \\ *corresponding author: tsia@hku.hk
}

\begin{abstract}
Nonlinearly generated broadband ultrafast laser have been increasingly utilized in many applications. However, traditional techniques of characterizing these sources lack the ability to observe the instantaneous features and transitory behaviours of both amplitude and phase. With the advent of the optical time stretch techniques, the instantaneous shotto-shot spectral intensity can be directly measured continuously at an unprecedentedly high speed. Meanwhile, the information of the real-time phase variation, which is carried by the frequency-time mapped spectral signal has yet been fully explored. We present a technique of experimentally measuring the spectral coherence dynamics of broadband pulsed sources. Our method relies on a delayed Young's type interferometer combined with optical time-stretch. We perform the proof-of-principle demonstrations of spectral coherence dynamics measurement on two sources: a supercontinuum source and a fiber ring buffered cavity source, both with a repetition rate of MHz. By employing the optical time stretch with a dispersive fiber, we directly map the spectral interference fringes of the delayed neighbouring pulses and obtain a sufficiently large ensemble of spectral interferograms with a real-time oscilloscope $(80 \mathrm{~Gb} / \mathrm{s} \mathrm{sampling}$ rate). This enables us to directly quantify the spectral coherence dynamics of the ultrafast sources with a temporal resolution down to microseconds. Having the ensemble of single-shot interferograms, we also further calculate the cross spectral coherence correlation matrices of these ultrafast sources. We anticipate that our technique provides a general approach for experimentally evaluating the spectral coherence dynamics of ultrafast laser generated by the nonlinear processes e.g. modulation instability, supercontinuum generation, and Kerr resonator.
\end{abstract}

Keywords: Spectral coherence, phase, ultrafast measurement, correlation map

\section{INTRODUCTION}

The palette of laser technology has significantly been enriched by the innovations in ultrafast optical pulse generation techniques, from frequency metrology, telecommunication, to optical imaging, biochemical spectroscopy [1-4]. Yet, current technologies lack the capability to fully dissect the complex dynamics of the pulse amplitudes and phases in realtime, continuously and with single-shot accuracy. On the fundamental physics front, such ability is invaluable to unravel the underlying mechanisms resulting in stochastic behaviors of the ultrafast pulse generation, which are closely linked to the intricate interactions between noise and nonlinear dynamical processes $[5,6]$. On the practical usage front, real-time monitoring and analysis of the shot-to-shot (at a rate of $\mathrm{MHz}$ or even $\mathrm{GHz}$ ) amplitude/phase fluctuation of the pulses are critical for designing highly stable and coherent pulsed lasers, which are stringently required by ultrafast applications [715]. Primarily limited by the acquisition speed of the spectrometers, classical spectral characterization techniques can only carry out ensemble averaged measurements, which mask the subtle properties of the shot-to-shot amplitude and phase dynamics. Meanwhile, nonlinear optical gating, and spectral shearing interferometry can be employed to retrieve the amplitude and phase of single pulses [16-21]. Recently, a new class of spectral characterization techniques, dubbed optical time-stretch, which enables shot-to-shot broadband pulse measurements, has recently caught considerable attention. The core concept is to map the instantaneous spectral amplitude (or intensity) of the pulses to the time domain through group velocity dispersion (GVD) [22,23]. With sufficient GVD, each single time-stretched pulse is essentially the replica of the frequency spectrum in time domain. Real-time single-shot spectral acquisition is thus simply achieved by high-speed digitizers.

While optical time stretch has opened a new avenue for spectrally-resolved single-shot characterization at an ultrahigh speed, majority of the existing effort focuses on intensity measurement and analysis [24-27]. Although phase retrieval

Real-time Measurements, Rogue Events, and Emerging Applications, edited by

Bahram Jalali, Sergei K. Turitsyn, Daniel R. Solli, John M. Dudley, Proc. of SPIE Vol. 9732,

97320C · (c) 2016 SPIE · CCC code: 0277-786X/16/\$18 - doi: 10.1117/12.2211969 
from single pulses is possible with iterative computational techniques [28], the phase accuracy is inherently limited by the time-bandwidth product, not to mention that the increased experimental complexity due to the required multiple/cascaded measurements [29]. To date, there is apparently a missing element in the optical time stretch technique that enables ultrafast broadband pulse-to-pulse phase variation measurement in real-time. For ultrafast pulse generation involving high nonlinearity and stochasticity, phase coherence is an important characterization metric. For instance, phase coherence of modulation instability [30] and rogue wave formation can be dramatically influenced by the presence of deterministic seeds/triggers [10,31,32]. In other nonlinear systems that involve solitonic dynamics [33], optical turbulence and chaotic behaviours [34,35], measurements of both spectral phase coherence as well as the intensity fluctuation in real-time have also long been absent, yet are expected to provide a more complete understanding of the underlying physics.

We here report the first experimental characterization of real-time spectral coherence with a temporal resolution down to microseconds based on optical time-stretch combined Young's type interferometry. Our approach hinges on high-speed evaluation of pulse-to-pulse phase instability based on the measured visibility of single-shot interferograms continuously-generated by interfering the neighbouring pulses. This is in contrast to the traditional (slow) Young's type interferometric methods in which only the degree of coherence over an ensemble of pulses can be evaluated. Also, our technique offers a new dimension to study the two-wavelength cross spectral coherence correlation-cross spectral density function (CDS) which is made possible only by the availability of single-shot interferograms.

\section{THEORY}

In this section, we outline the procedure the two-frequency cross spectral density function (CDS) of the supercontinuum generation (SC) retrieved from the intensity of the ensemble interferograms. We firstly numerically simulate 1000 runs of optical pulse (Gaussian pulse profile) propagated along a $20 \mathrm{~m}$ photonic crystal fiber (PCF) with the nonlinear Schrödinger equation (NLSE)

$$
\frac{\partial A}{\partial z}=\sum_{k \geq 2} \frac{i^{k}}{k !} \beta_{k} \frac{\partial^{k} A}{\partial t^{k}}+i \gamma A(z, t) \int_{-\infty}^{+\infty} R\left(t^{\prime}\right)\left|A\left(z, t-t^{\prime}\right)\right|^{2} d t^{\prime},
$$

where $A(z, t)$ is the envelope of the optical field, $\beta_{k}$ are the dispersion coefficient, $\gamma$ is the nonlinear coefficient of the PCF, and $R(t)$ is the Raman response function contained the instantaneous and Raman delay attributed to the nonlinearity. Please refer to Ref. [36] for the details of the simulation parameters. SC generated in two different pumping regimes-ps pumping ( $1.2 \mathrm{ps})$ and fs pumping $(400 \mathrm{fs})$ will be investigated. The simulated output spectra at the end of the PCF for these two scenarios are shown in Figure 1.
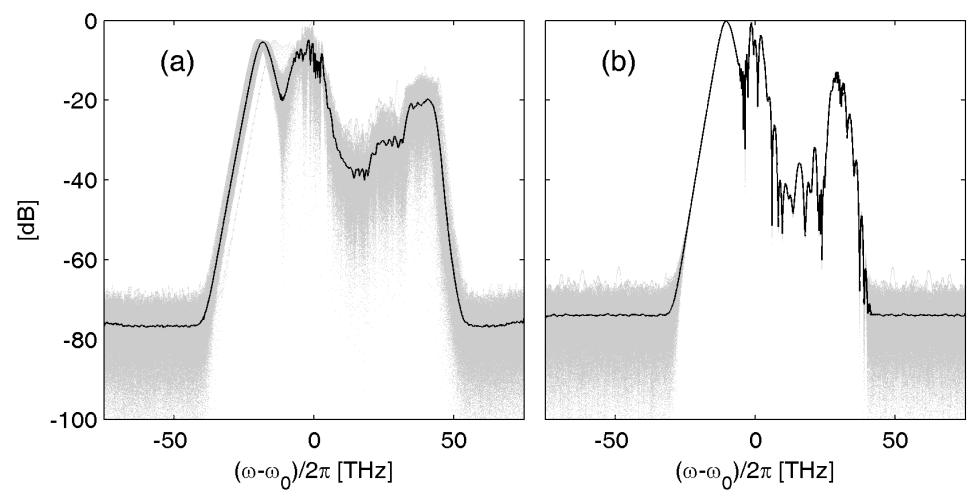

Figure 1. The ensemble of simulated SC of 1000 runs. (a) and (b) are the SC generated by ps-pumping and fs-pumping, respectively. The gray dots are the ensemble of the spectra, and the black curves are the spectra of the ensemble average.

We wish to establish the two-frequency correlation functions of the optical field of the second order theory based on this ensemble of spectral fringes, which also corresponds to what we can experimentally measure. According to Ref. [37], normalized form of the two-frequency cross-spectral density (CSD) function is defined as 


$$
\mu\left(\omega_{1}, \omega_{2}\right)=\frac{\left\langle\widetilde{E}^{*}\left(\omega_{1}\right) \widetilde{E}\left(\omega_{2}\right)\right\rangle}{\sqrt{\left\langle\left|\widetilde{E}\left(\omega_{1}\right)\right|^{2}\right\rangle\left\langle\left.\widetilde{E}\left(\omega_{2}\right)\right|^{2}\right\rangle}},
$$

where $\widetilde{E}(\omega)$ is the complex spectrum of the Fourier transform of the envelope $A(t)$ of the 1000 runs, and \langle\rangle denotes the ensemble average. In our system, what we measure is the ensemble of the spectral fringes which individually can be expressed as

$$
I(\omega, \tau)=\left|\widetilde{E}_{1}(\omega, 0)+\widetilde{E}_{2}(\omega, \tau)\right|^{2}=\left|\widetilde{E}_{1}(\omega, 0)\right|^{2}+\left|\widetilde{E}_{2}(\omega, \tau)\right|^{2}+2 \Gamma_{12}(\omega, \tau),
$$

where $\tau=\left(z_{2}-z_{1}\right) / c$ is the time mismatch between the neighbouring pulses, and the third term is the spectral interferogram (also known as AC component)

$$
\Gamma_{12}(\omega, \tau)=\mathfrak{R}\left\{\widetilde{E}_{1}^{*}(\omega) \widetilde{E}_{2}(\omega, \tau)\right\}=\left|\widetilde{E}_{1}(\omega)\right|\left|\widetilde{E}_{2}(\omega)\right| \cos [\omega \tau-\varphi(\omega)],
$$

where $\varphi(\omega)$ is the instantaneous phase of each frequency component of the interferogram, and the normalized individual spectral interferogram is

$$
\gamma_{12}(\omega, \tau)=\frac{\Gamma_{12}(\omega, \tau)}{\left|\widetilde{E}_{1}(\omega)\right|\left|\widetilde{E}_{2}(\omega)\right|}=\cos [\omega \tau-\varphi(\omega)],
$$

After decoupling the spectral intensity from the interferogram, it can be seen that the spectral coherence inherently lives in the instantaneous phase term $\varphi(\omega)$. It has been well known that the $\left|g_{12}^{(1)}\right|$ can be directly derived by taking the amplitude of the mean normalized interferograms as

$$
\left|g_{12}^{(1)}(\omega)\right|=\left\|\left\langle\gamma_{12}(\omega, \tau)\right\rangle\right\| \approx\left|\frac{\widetilde{E}_{1}^{*}(\omega) \widetilde{E}_{2}(\omega, \tau)}{\sqrt{\left\langle\left|\widetilde{E}_{1}(\omega)\right|^{2}\right\rangle\left\langle\left.\widetilde{E}_{2}(\omega)\right|^{2}\right\rangle}}\right| \text {, for } \omega \tau>>1,
$$

where \|\| denotes as taking the amplitude here, and $\omega \tau>>1$ is the assumption that the spectral intensity envelope varies slowly with respect to the fringes period. Similarly, without further normalization, the recovered CSD function can be expressed as

$$
\hat{\mu}\left(\omega_{1}, \omega_{2}\right)=\sqrt{\left\|\left\langle\gamma_{12}\left(\omega_{1}, \tau\right) \gamma_{12}\left(\omega_{2}, \tau\right)\right\rangle\right\|} .
$$

To demonstrate how to recover the CSD function with the ensemble of spectral interferograms, we compute $\hat{\mu}\left(\omega_{1}, \omega_{2}\right)$ using the ensemble of the intensity profiles of the spectral fringes $I_{n}(\omega, \tau)=\mid F\left[A_{1}(t)+A_{2}(t+\tau)\right]^{2}$ obtained from the 1000 runs of SC, and compare the directly calculated CSD function $\mu\left(\omega_{1}, \omega_{2}\right)$ with the full field spectra $\widetilde{E}_{n}(\omega)$ using Eq. (2). Following the steps indicated in Figure 2, we plot in Figure 2 (a) and (b), the ensemble of the spectral interferograms of SC generated by ps-pumping, and the cross-correlated interferogram $\left\langle\gamma_{12}\left(\omega_{1}, \tau\right) \gamma_{12}\left(\omega_{2}, \tau\right)\right\rangle$ based on $\gamma_{12}(\omega, \tau)$, respectively. Finally, we calculate the recovered CSD function $\hat{\mu}\left(\omega_{1}, \omega_{2}\right)$ based on the amplitude of the cross-correlation map of the interferogram, and plot in Figure 2 (d). By defining a proper moving window over the map, the amplitude of the correlation of interferogram can be simply extracted from the amplitude of the frequency component of the 2-D FFT that corresponds to frequency of the spectral fringes. In order to suppress amplitude contribution of the diagonal line (i.e. self-correlation) of the correlation map of the interferogram, the frequency component that is 
orthogonal to the direction of the diagonal line in the 2-D FFT map has been chosen. Compared with the normalized CSD function $\mu\left(\omega_{1}, \omega_{2}\right)$ that shown in Figure 2 (c), it can be clearly seen that the recovered CSD $\hat{\mu}\left(\omega_{1}, \omega_{2}\right)$ can highly restore most of the feature of the CSD $\mu\left(\omega_{1}, \omega_{2}\right)$.
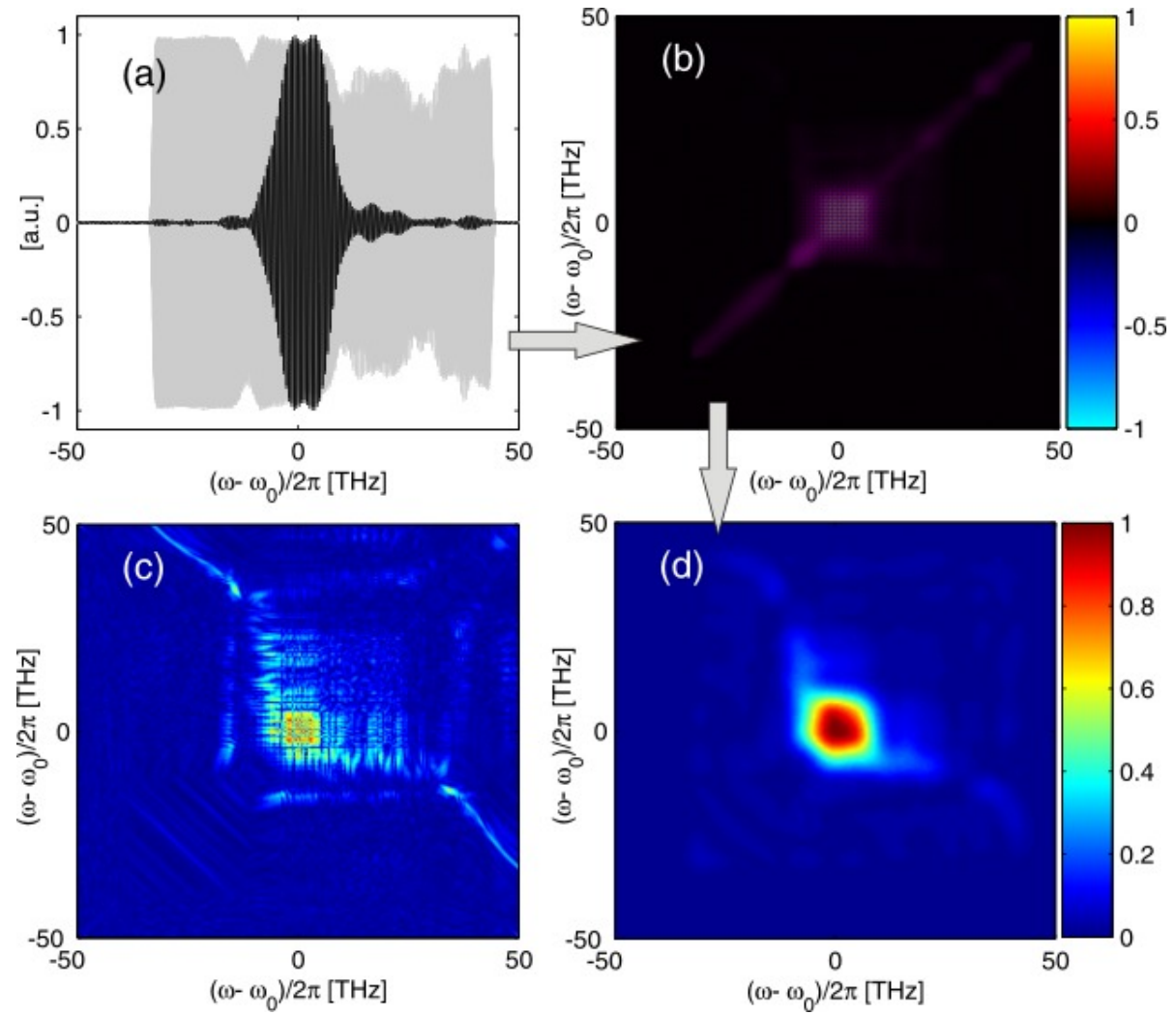

Figure 2. (a) The ensemble of the normalized spectral interferograms $\gamma_{12}(\omega, \tau)$ (grey curves) and the ensemble average of the normalized $\left\langle\gamma_{12}(\omega, \tau)\right\rangle$ (black curve). (b) The cross correlated spectral interferogram calculated based on Eq. (7). (c) The CSD function $\mu\left(\omega_{1}, \omega_{2}\right)$ of the ps-pumping SC calculated using Eq. (2). (d) The recovered CSD function $\hat{\mu}\left(\omega_{1}, \omega_{2}\right)$ based on the amplitude of the cross correlated spectral interferogram in (b).
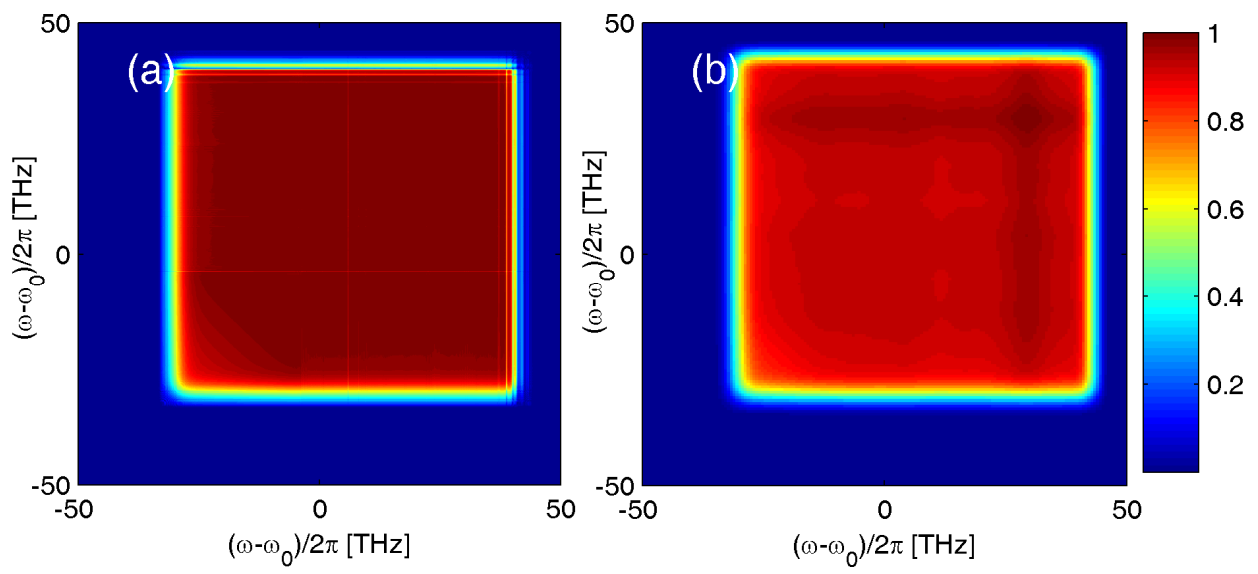

Figure 3. (a) The CSD function $\mu\left(\omega_{1}, \omega_{2}\right)$ of the SC generated by fs-pumping (b) The recovered CSD function $\hat{\mu}\left(\omega_{1}, \omega_{2}\right)$ of fs-pumping SC. 
The loss of fast variation feature in the CSD function is attributed to the overlapping between the fringe period and the fast spectral variation. Experimentally, this can be simply overcome by increasing the total dispersion of the time-stretch process and increasing the bandwidth of the detection system. To further validate the recovered CSD function, we also plot in Figure 3 (a) $\mu\left(\omega_{1}, \omega_{2}\right)$ and $\hat{\mu}\left(\omega_{1}, \omega_{2}\right)$ of the SC generated by fs pumping. As we expected, Figure 3 (a) shows that the SC generated by fs pumping is highly coherent across the entire CSD function, and the recovered CSD function in Figure 3 (b) can well restore the feature of the CSD function.

\section{EXPERIMENTAL SETUP}

In contrast to the conventional configuration adopted for the static spectral coherence measurement [17-21], our experimental setup consists not only a free-space Michelson interferometer with one of the arms delayed by one period of the pulse source, but also a time-stretch module after the interferometer-the enabling element for ultrafast spectral coherence measurement as shown in Figure 4. The interfered pulses are time-stretched by a $10.7 \mathrm{~km}$-long dispersion compensation fiber (DCF) with a total GVD of $-932 \mathrm{ps} / \mathrm{nm}$ at $1563.8 \mathrm{~nm}$. The stretched temporal fringes, i.e. the singleshot interferograms, are then detected by a $10 \mathrm{GHz}$ photodiode and a real-time oscilloscope $(80 \mathrm{GSa} / \mathrm{sec})$. The fringe period of the interferogram is adjusted such that the system has enough temporal resolution to resolve the fringes and at the same time, the fringe density over the spectral envelope is sufficiently high to retain the overall spectral feature.

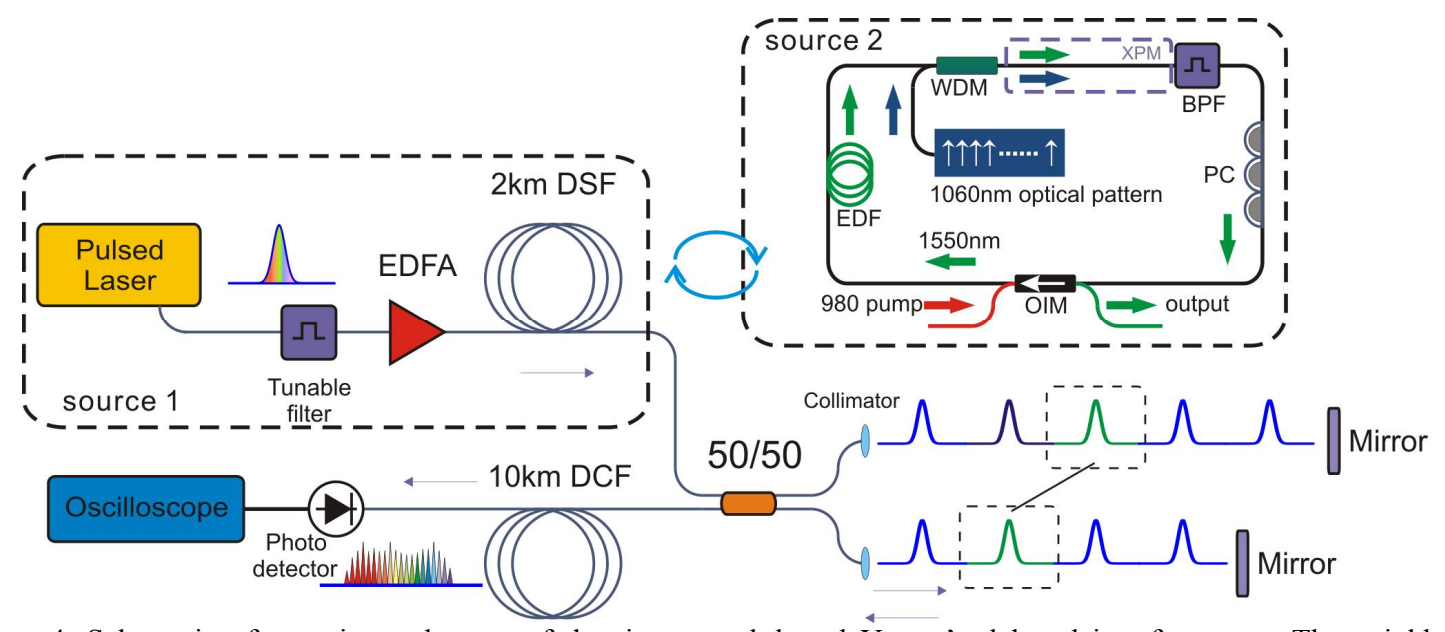

Figure 4. Schematic of experimental setup of the time-stretch-based Young's delayed interferometer. The neighboring pulses are combined and interfered. Then the interfered pulses go through a 10-km long DCF after which the single-shot interferograms are mapped into the time-domain. 50/50 refers to the beam splitter with 50:50 splitting ratio. EDFA: Erbium-doped fibre amplifier, DSF: dispersion shifted fiber, DCF: dispersion compensation fiber, OIM: optical integrated module, PC: polarization controller, BPF: bandpass filter, EDF: Erbium-doped fibre,WDM: wavelength division multiplexer.

\subsection{Supercontinuum source}

The SC is generated by launching a fiber mode-locked laser pulsed pump (centred at $1563.8 \mathrm{~nm}$ with a repetition rate of $46 \mathrm{MHz}$ ) into a 2-km long dispersion shifted fiber (DSF) with the zero dispersion wavelength around $1550 \mathrm{~nm}$. The linewidth of the pump pulse is controlled by a linewidth tunable filter. The filtered pump pulse is pre-amplified by an erbium doped fiber amplifier (EDFA) before the DSF. Using the tunable filter, we are able to manipulate SC in two different pumping regimes: (i) picosecond (2.2 ps) and femtosecond (780 fs) pulsed pump.

\subsection{Buffered cavity laser}

Our second type of light sources used for the coherence measurement is optical buffered pulses which are generated by an all-fiber ring cavity buffer with an external addressing mode-locked laser, as shown in Figure 4. For more details about this cavity buffer and addressing laser, please refer to Ref. [7, 38]. The inline polarization controller (PC) before optical integrated module (OIM) was employed to adjust the state of polarization of the optical field inside the all-fiber ring cavity, which together with the polarization-sensitive OIM sustains the buffered pulse oscillation after excited by an external pulse laser with a wavelength far beyond the lasing wavelength of the fiber cavity, i.e. promotes the artificially 
saturable absorber effect $[39,40]$. The buffer cavity was adjusted to emit $\mathrm{CW}$ wave around $1558 \mathrm{~nm}$ without the addressing pulses. The CW output from the EDF would be combined with the addressing signal pulse from an external pulse laser at $1060 \mathrm{~nm}$ for the excitation through a fiber-based WDM. The $1558 \mathrm{~nm}$ CW wave would be "modulated", via XPM, to be the pulse pattern the same as that of the addressing beam [41, 42]. For the purpose of testing the coherence of the buffered pulses, we control the coherence of the buffered pulse by adjusting the synchronization between the lengths of the buffered cavity and addressing pulsed laser cavity.

\section{EXPERIMENTAL RESULTS}

\subsection{Spectral coherence measurements by OSA and time-stretch interferometry}

We compare the spectral coherence measured by our technique and by traditional ensemble average method with optical spectrum analyzer.
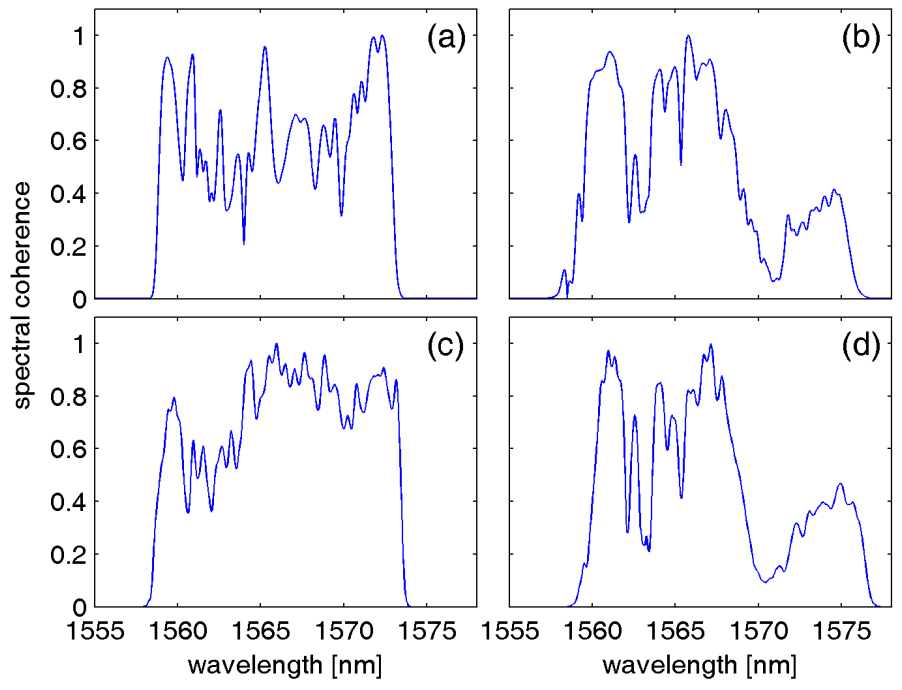

Figure 5. (a) and (b) are, respectively, the spectral coherence of fs-pumping and ps-pumping SC generated measured by OSA. (c) and (d) are, respectively, the spectral coherence of fs-pumping and ps-pumping SC generated measured by OSA. by the interferometry time-stretch setup.

We plot in Figure 5 (c) and (d) the experimentally measured spectral coherence of the fs and ps pumping SC which is originally from Figure 5 (b) and (d) in the main text. We also measure the spectral coherence of the SC sources using an optical spectrum analyzer, and plot them in Figure 5 (a) and (b). As we can see, the spectral coherence measurements by OSA and time-stretch interferometry agree well with each other. We attribute the slight discrepancy between these two techniques to the long term stability of the interferometer setup, as mechanical vibration has a larger influence on the OSA measurement in which the acquisition time is in second order.

\subsection{Spectral coherence measurements of fs-pumped and ps-pumped SC generation}

We first demonstrate the capability of our technique to observe the conventional static SC spectral coherence. Thanks to the time-stretch technique, we capture 500 single-shot interferograms for both pumping scenarios at an ultrafast rate of $46 \mathrm{MHz}$ (see Figure 6 (a) and (c)). Note that each spectral interferogram is intrinsically generated by the interferometer, and are transformed into a serial temporal interferograms by optical time stretch. Again, we here focus on the degree of spectral coherence influenced by the amplitude and phase stability which can be evaluated by the fringe visibility of the interferograms. 

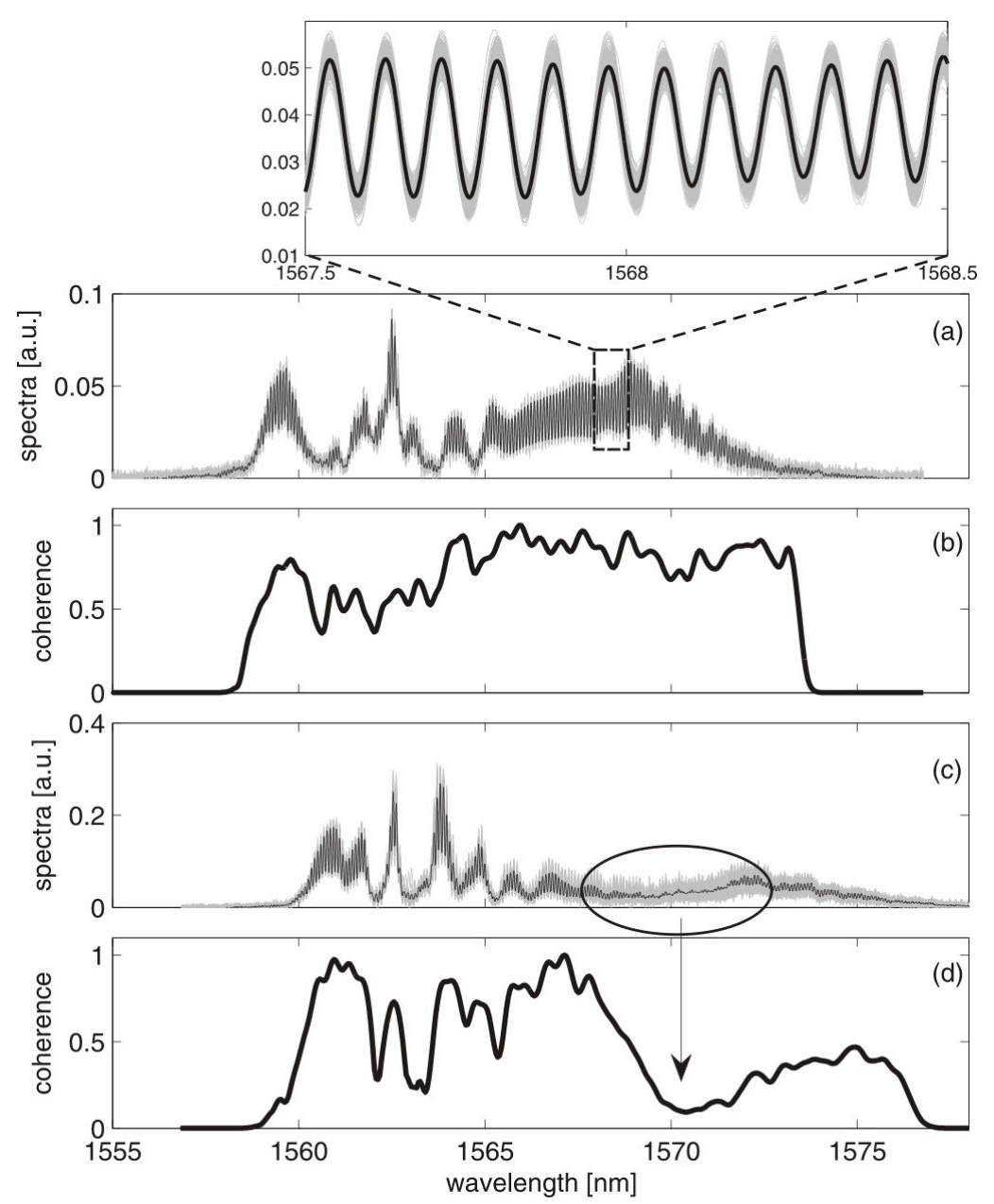

Figure 6. Measured spectral coherence by time-stretch interferometry. (a) and (c) are the experimentally observed 500 consecutive single-shot interferograms (the overlapped gray interferograms) of the SC generated by the femtosecond (780 fs) and picosecond (2.2 ps) pumps, respectively. They are captured at a rate of $46 \mathrm{MHz}$. The black interferograms are the averaged interferograms. (b) and (d) are the corresponding spectral coherence.

As expected, in the case of fs pumping regime, in which the spectral broadening is dominated by self-phase modulation (SPM) and subsequent soliton fission. High spectral coherence can be clearly observed over the entire spectrum (Figure 6 (b)). On the contrary, the SC generated by ps pumping (Figure 6 (d)) is generally less coherent in the long wavelength side, especially from $1568 \mathrm{~nm}$ to $1572 \mathrm{~nm}$ (indicated by the circle in Figure 6 (c), with the corresponding spectral coherence pointed by arrow in Figure 6 (d)). We attribute this low coherence region to the process of the soliton formation which onsets from the noise-driven modulation instability effects when pumping a ps-pump [6,44]. Note that as the entire ensemble of interferograms is captured well within millisecond in the experiments reported in this work. Any bulk optomechanical vibration and drifting of the system is expected to be in the low frequency range $(<<\mathrm{kHz})$, which is thus negligible in our measurements. For long-term continuous measurements, feedback stabilization of the mechanical drift can be implemented to ensure long-term mechanical stability of the interferometer.

Apart from evaluating the static coherence, we also show that time-stretch-based Young's interferometry uniquely enables a more comprehensive, yet underexplored approach to experimentally study the CSD function. Again, we leverage the ensemble of the single-shot interferograms $\gamma_{12}(\lambda)$ to compute the recovered CSD function $\hat{\mu}\left(\lambda_{1}, \lambda_{2}\right)$. 
(a)

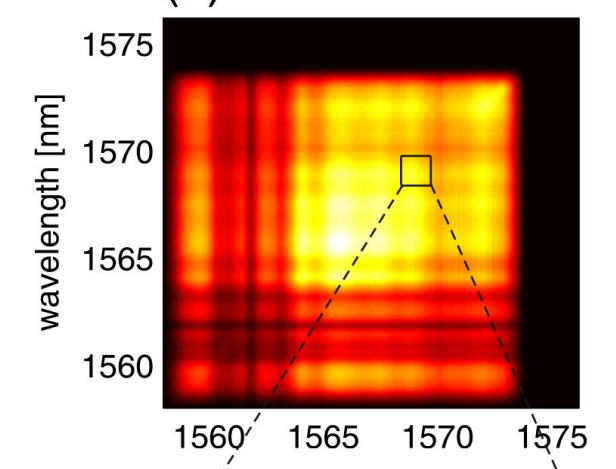

(b)

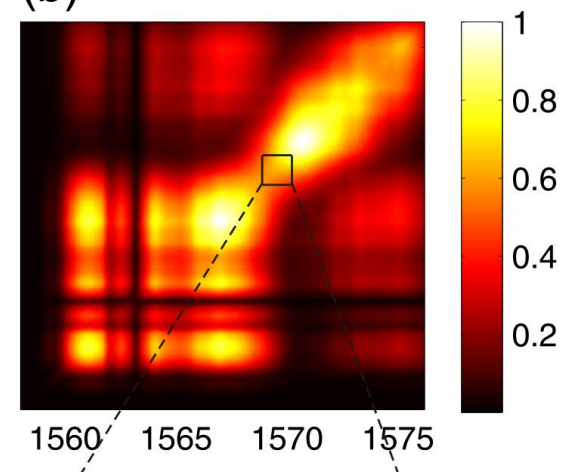

(d́)

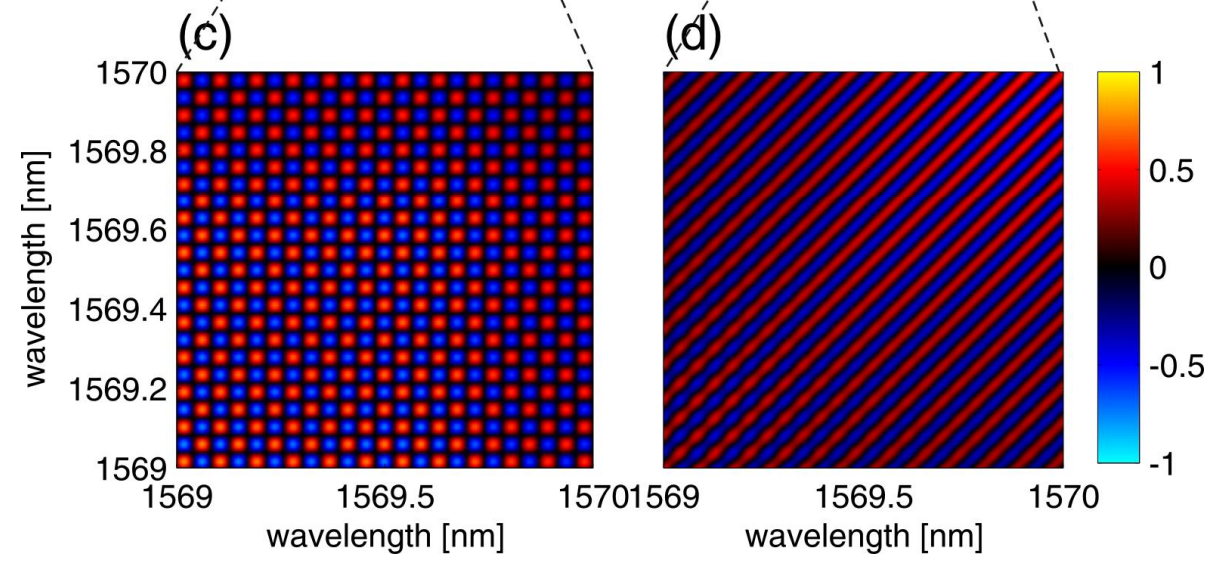

Figure 7. Cross-spectral function (CSD) derived from time-stretch interferometry. (a) and (b) are the recovered CSD functions $\hat{\mu}\left(\lambda_{1}, \lambda_{2}\right)$ of SC sources in femtosecond and picosecond pumping regimes, respectively. (c) and (d) and crosscorrelation maps of the interferograms $\left\langle\gamma_{12}\left(\lambda_{1}\right) \gamma_{12}\left(\lambda_{2}\right)\right\rangle$ referring to the highlighted regions in (a) and (b), respectively. 500 single-shot interferograms are taken for the calculation.

Figure 7 (a) and (b) show the recovered CSD function $\hat{\mu}\left(\lambda_{1}, \lambda_{2}\right)$ of for both ps and fs pumping cases. In the case of fspumped SC, it displays a high degree of coherence correlation across the entire spectrum, manifested as an almost uniform square with the value close to unity in the map (Figure 7 (a)). It means that the spectral components are highly correlated in this ensemble, and this is consistent to the fact that the fs-pumped SC generation is primarily dominated by the deterministic spectral broadening processes. In contrast, the correlation map of the ps-pumped SC only has the high coherence correlation mainly along the diagonal line (corresponding to the self-correlation of each wavelength component), leaving a relatively low coherence along the two sides of the diagonal line of the map. This means a general incoherence and low spectral correlation across the long wavelength of SC, which is attributed to the noise-driven MI and the subsequent spectral broadening in the ps-pumped SC. Note that the map can reveal a finite spectral correlation between the pump and the soliton regions (see the top left (or bottom right) region in the map (Figure 7 (b)).

More interestingly, when we study the correlation map of the normalized spectral interferograms $\left\langle\gamma_{12}\left(\lambda_{1}\right) \gamma_{12}\left(\lambda_{2}\right)\right\rangle$ instead of $\hat{\mu}\left(\lambda_{1}, \lambda_{2}\right)$, we observe that the fs-pumped SC exhibits high visibility with a sharp square-grid pattern in the long wavelength side (close to the diagonal line) whereas the ps-pumped SC shows the sheared grids or even diagonal strips (Figure 7 (c) and(d)). The square grids are a result of the well-defined change in visibility across the interferograms (i.e. the magnitudes of maxima and minima of the interferograms vary collectively). Shearing of the grid represents not only the change in visibility, but also the spectral phase jitter across the spectrum. This follows the similar jittering argument used in intensity cross-correlation analysis $[25,45]$. The difference is that we focus on the phase jitter as we consider the spectral interferograms instead of the spectral amplitudes. Therefore, sheared grids in Figure 7 (d) can be interpreted as the spectral phase jitter of the new frequency components generated by the Raman induced self-frequency 
shift. This spectral phase jitter effectively leads to the low spectral coherence in the ensemble, as indicated by the circle in Figure 7 (b).

\subsection{Spectral coherence dynamics measurement}

To further demonstrate the capability of high-speed spectral coherence variation measurement enabled by our technique, we study another type of pulsed laser source-generating optical buffered pulses within an all-fiber ring cavity, which is modulated ("addressed") with an external mode-locked laser [38]. It is proven to be an effective all-optical approach to actively manipulate the intra-cavity multipulses in mode-locked lasers, which commonly emerge from the combination of nonlinear mechanisms in the presence of noise, e.g. spectral filtering effect, wave breaking effect and soliton peak clamping effect. As far as stable mode-locked laser development, buffered light source is of great value to control pulsing in the laser cavity. In brief, the cavity originally emits continuous wave at $1558 \mathrm{~nm}$ in the absence of the addressing pulses. It in turn sustains buffered pulse oscillation when modulated, via cross-phase modulation, by an external addressing pulsed laser with a large wavelength-detune (at $1060 \mathrm{~nm}$ in our case)-resembling as a saturable absorber effect $[39,40]$. As the resultant modulated pattern is replicated from the addressing laser [41, 42] with an ultrawideband wavelength conversion of $\sim 500 \mathrm{~nm}$, this scheme is particularly useful for all-optical delay lines [46].

It is expected that the coherence of the buffered pulses is strongly influenced the level of synchronization between the buffered cavity and the external addressing pulsed laser cavity, which can be manipulated by the cavity lengths. We thus measure in real-time the spectral coherence evolution of the buffered pulses in two scenarios in which (i) the cavities are perfectly synchronized; (ii) the cavities are partially desynchronized (Figure 8 (a) and (b)). Each measurement consists of 8192 single-shot interferograms over a time windows of $300 \mu \mathrm{s}$. We compute spectral coherence evolution based on a moving ensemble with 256 interferograms. With an overlapped size between the neighboring ensembles of 32 , the equivalent temporal resolution of the real-time spectral coherence measurement is as short as $1.2 \mu \mathrm{s}$. This implies the system is able to probe fast spectral coherence variation close to the range of $\mathrm{MHz}$.

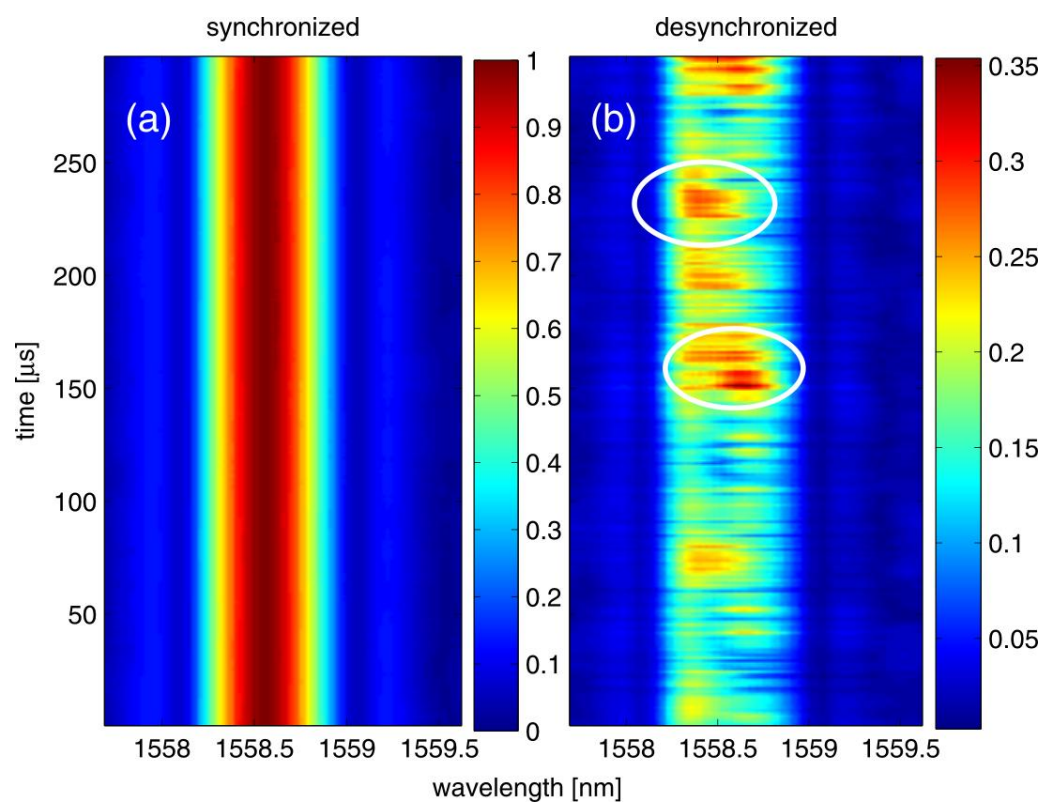

Figure 8. Time evolution of spectral coherence of buffered cavity. (a) Perfectly synchronized injection and (b) partially desynchronized injection. Highlighted circles show the "slow" localized structures embedded in the stochastic variation of spectral coherence.

Clearly, the perfectly synchronized buffered pulses possess a higher and more stable spectral coherence than the partially desynchronized pulses. The low spectral coherence of the desynchronized pulses is mainly attributed to "modulation" timing jitter between addressing pulses and the buffered intracavity pulses. The ultrashort time jittering manifests itself as the spectral phase shifts and consequently low spectral fringes contrast of an ensemble. While the spectral coherence of the desynchronized pulses exhibits a large amount of fluctuations along the evolving time, our technique enables us to identify an interesting feature that moderate coherence $(\sim 0.35)$ is localized over both spectral domain (within $0.5 \mathrm{~nm})$ as well as and "slow" temporal evolution domain (over $20 \mu \mathrm{s}$ ). (see the highlighted circles of Figure 8 (b)). These local 
structures appear in a rather stochastic manner, yet tend to persist for hundreds of cavity round-trips. To the best of our knowledge, such real-time, fast, and non-repetitive coherence variations have not be experimentally revealed by any existing (slow) spectral measurement techniques. We note that similar stochastic, localized radiations have also been observed in the partially mode-locking scenarios [35]. While it is yet to be fully understood, the availability of both spectral coherence and intensity information in real-time could bring new insights as well as experimental methods in understanding the complex (and high-speed) nonlinear processes. Notably, one could leverage on the arbitrary addressing patterns for flexible and high-precision synchronized coherence measurements, such as pump-probe studies, seeding analysis of stochastic processes in highly nonlinear systems (e.g. SC generation, partially mode-locking, and chaotic lasers).

\section{DISCUSSION}

Taking the advantage of its capability to quantify the high-speed broadband spectral coherence in real-time, single-shot time-stretch interferometry is particularly useful for probing the stochastic or noise-sensitive systems in which the amplitude and phase fluctuation dynamics are in short-time scale. These systems have been attracting considerable interest from the fundamental physics point of view. Notable examples include complex nonlinear intracavity dynamics in mode-locked laser, that is recently known to lead to chaotic or noise-like radiation, e.g. optical turbulence [47-49]; stochastic behaviors in SC generation, e.g. modulation instability, the subsequent soliton fission and rogue wave formations [50]. Indeed, our ability to experimentally probe these dynamics has been limited to the spectral intensity domain, which is not exhaustive. The technique demonstrated here enables comprehensive ultrafast pulse characterization-the coherence spectra at a sub-microsecond time scale in real-time. More importantly, it allows us to experimentally evaluate the second-order spectral coherence, i.e. CSD, thanks to the availability of single-shot interferograms-another feature generally missing in the current techniques.

Given these capabilities, the technique could bring new perspectives in experimental investigations of those intricate nonlinear dynamics which have only been possible with numerical simulations. For instance, many of these effects are sensitive to input noise or other external perturbation. Hence, one can implement synchronized pump-probe, feedback, seeding/triggering measurements in which the seed/trigger can be arbitrarily engineered (in terms of temporal/spectral width, power, and spatio-temporal patterns) to probe and even manipulate those phenomena. Using time-stretch interferometry can readily reveal the corresponding real-time rapid responses in both spectral coherence as well as intensity variations. More practically, deeper understanding of these underlying processes could provide insight into how to manipulate them and thus pave the way for developing highly stable (shot-to-shot), low-noise pulsed lasers, especially valuable for applications requiring high temporal stability, such as ultrafast imaging and spectroscopy [2]. Note that seeded/triggered SC has already been studied with time-stretch spectral intensity measurements, especially in the context of "stimulating" or "inhibiting" rogue wave formation [10, 51], which give clues for SC enhancement or suppression [27]. Again, the dynamics of spectral coherence have not been fully characterized in many of these highly nonlinear SC generation processes.

\section{CONCLUSION}

In conclusion, we have presented a technique, based on time-stretch Young's interferometry, which uniquely provides access to single-shot interferograms from which real-time spectral coherence dynamics of ultrafast laser sources can be evaluated experimentally with a temporal resolution down to the microsecond scale. Having validated the measured spectral coherence by comparing it with the common $g_{12}^{(1)}$ definition, we have demonstrated that the technique not only can quantify the static coherence (as demonstrated with the SC generation), but also unveil the high-speed spectral coherence fluctuation (as exemplified by a buffered cavity pulsed source) on the order of MHz. Furthermore, the large ensemble acquired by the single-shot measurement provides us sufficient information to further reconstruct the CSD function that cannot be achieved by the traditional coherence measurement techniques. The fact that our technique can provide a handful and comprehensive toolset to gain insight into the spectral coherence dynamics of a plethora of optical ultrafast lasers as well as nonlinear optical effects, such as rogue wave formation [5], modulation instability [30], solitonic dynamics [33], Kerr resonator [42], as well as optical turbulence and chaotic behaviors in pulse laser [34, 35]. 


\section{REFERENCES}

[1] Holzwarth, R., Udem, T., Hänsch, T. W. et al., "Optical Frequency Synthesizer for Precision Spectroscopy," Physical Review Letters 85(11), 2264-2267 (2000).

[2] Goda, K., Tsia, K. K., and Jalali, B., "Serial time-encoded amplified imaging for real-time observation of fast dynamic phenomena," Nature 458(7242), 1145-1149 (2009).

[3] Masters, B. R., and So, P. T. C., [Handbook of biomedical nonlinear optical microscopy] Oxford University Press, New York(2008).

[4] Fard, A. M., Gupta, S., and Jalali, B., "Photonic time-stretch digitizer and its extension to real-time spectroscopy and imaging," Laser \& Photonics Reviews 7(2), 207-263 (2013).

[5] Solli, D. R., Ropers, C., Koonath, P. et al., "Optical rogue waves," Nature 450(7172), 1054-1057 (2007).

[6] Dudley, J. M., Genty, G., and Coen, S., "Supercontinuum generation in photonic crystal fiber," Reviews of Modern Physics 78(4), 1135-1184 (2006).

[7] Wei, X., Lau, A. K. S., Wong, T. T. W. et al., "Coherent Laser Source for High Frame-Rate Optical TimeStretch Microscopy at 1.0 um," Selected Topics in Quantum Electronics IEEE Journal 20(5), 384-389 (2014).

[8] Cheung, K. K. Y., Zhang, C., Zhou, Y. et al., "Manipulating supercontinuum generation by minute continuous wave," Opt. Lett. 36(2), 160-162 (2011).

[9] Zhang, C., Qiu, Y., Zhu, R. et al., "Serial time-encoded amplified microscopy (STEAM) based on a stabilized picosecond supercontinuum source," Opt. Express 19(17), 15810-15816 (2011).

[10] Solli, D. R., Ropers, C., and Jalali, B., "Active Control of Rogue Waves for Stimulated Supercontinuum Generation," Physical Review Letters 101(23), 233902 (2008).

[11] Solli, D. R., Jalali, B., and Ropers, C., "Seeded Supercontinuum Generation with Optical Parametric DownConversion," Physical Review Letters 105(23), 233902 (2010).

[12] Xu, J., Wei, X., Yu, L. et al., "High-performance multi-megahertz optical coherence tomography based on amplified optical time-stretch," Biomed. Opt. Express 6(4), 1340-1350 (2015).

[13] Ma, J., Yuan, P., Wang, J. et al., "Spatiotemporal noise characterization for chirped-pulse amplification systems," Nat Commun 6, 6192 (2015).

[14] Wong, T. T. W., Lau, A. K. S., Wong, K. K. Y. et al., "Optical time-stretch confocal microscopy at $1 \mu$ m," Opt. Lett. 37(16), 3330-3332 (2012).

[15] Wong, T. T. W., Lau, A. K. S., Ho, K. K. Y. et al., "Asymmetric-detection time-stretch optical microscopy (ATOM) for ultrafast high-contrast cellular imaging in flow," Scientific Reports 4, 3656 (2014).

[16] Wong, T. C., Rhodes, M., and Trebino, R., "Single-shot measurement of the complete temporal intensity and phase of supercontinuum," Optica 1(2), 119-124 (2014).

[17] Gu, X., Kimmel, M., Shreenath, A. et al., "Experimental studies of the coherence of microstructure-fiber supercontinuum," Opt. Express 11(21), 2697-2703 (2003).

[18] Baum, P., Lochbrunner, S., Piel, J. et al., "Phase-coherent generation of tunable visible femtosecond pulses," Opt. Lett. 28(3), 185-187 (2003).

[19]Lu, F., and Knox, W., "Generation of a broadband continuum with high spectral coherence in tapered singlemode optical fibers," Opt. Express 12(2), 347-353 (2004).

[20] Nicholson, J., and Yan, M., "Cross-coherence measurements of supercontinua generated in highly-nonlinear, dispersion shifted fiber at $1550 \mathrm{~nm}$, , Opt. Express 12(4), 679-688 (2004).

[21]Zeylikovich, I., Kartazaev, V., and Alfano, R. R., "Spectral, temporal, and coherence properties of supercontinuum generation in microstructure fiber," J. Opt. Soc. Am. B 22(7), 1453-1460 (2005).

[22] Goda, K., Solli, D. R., Tsia, K. K. et al., "Theory of amplified dispersive Fourier transformation," Phys Rev A 80(4), 043821 (2009).

[23] Goda, K., and Jalali, B., "Dispersive Fourier transformation for fast continuous single-shot measurements," Nat Photon 7(2), 102-112 (2013).

[24] Wetzel, B., Stefani, A., Larger, L. et al., "Real-time full bandwidth measurement of spectral noise in supercontinuum generation," Sci. Rep. 2, 882 (2012).

[25] Godin, T., Wetzel, B., Sylvestre, T. et al., "Real time noise and wavelength correlations in octave-spanning supercontinuum generation," Opt. Express 21(15), 18452-18460 (2013).

[26] Runge, A. F. J., Aguergaray, C., Broderick, N. G. R. et al., "Coherence and shot-to-shot spectral fluctuations in noise-like ultrafast fiber lasers," Opt. Lett. 38(21), 4327-4330 (2013). 
[27]Ren, Z., Xu, Y., Qiu, Y. et al., "Spectrally-resolved statistical characterization of seeded supercontinuum suppression using optical time-stretch," Opt. Express 22(10), 11849-11860 (2014).

[28] Solli, D. R., Gupta, S., and Jalali, B., "Optical phase recovery in the dispersive Fourier transform," Applied Physics Letters 95(23), 231108 (2009).

[29] Xu, Y., Ren, Z., Wong, K. K. Y. et al., "Overcoming the limitation of phase retrieval using Gerchberg Saxtonlike algorithm in optical fiber time-stretch systems," Opt. Lett. 40(15), 3595-3598 (2015).

[30] Solli, D. R., Ropers, C., and Jalali, B., "Measuring single-shot modulation instability and supercontinuum spectra at megahertz rates," Nonlinearity 26(3), R85 (2013).

[31] Sørensen, S. T., Larsen, C., Møller, U. et al., "The role of phase coherence in seeded supercontinuum generation," Opt. Express 20(20), 22886-22894 (2012).

[32] Wei, X., Zhang, C., Xu, S. et al., "Effect of the CW-Seed's Linewidth on the Seeded Generation of Supercontinuum," Selected Topics in Quantum Electronics IEEE Journal 20(5), 605-611 (2014).

[33] Erkintalo, M., Genty, G., Wetzel, B. et al., "Akhmediev breather evolution in optical fiber for realistic initial conditions," Physics Letters A 375(19), 2029-2034 (2011).

[34] Turitsyna, E. G., Smirnov, S. V., SugavanamS et al., "The laminar-turbulent transition in a fibre laser," Nat Photon 7(10), 783-786 (2013).

[35] Churkin, D. V., Sugavanam, S., Tarasov, N. et al., "Stochasticity, periodicity and localized light structures in partially mode-locked fibre lasers," Nat Commun 6, 7004 (2015).

[36] Qiu, Y., Xu, Y. Q., Wong, K. K. Y. et al., "Enhanced supercontinuum generation in the normal dispersion pumping regime by seeded dispersive wave emission and stimulated Raman scattering," Optics Communications 325(0), 28-34 (2014).

[37] Genty, G., Surakka, M., Turunen, J. et al., "Complete characterization of supercontinuum coherence," J. Opt. Soc. Am. B 28(9), 2301-2309 (2011).

[38] Wei, X., Xu, Y., Tan, S. et al., "Pulsing Manipulation in a $1.55 \mu \mathrm{m}$ Mode-Locked Fiber Laser by a $1.0 \mu \mathrm{m}$ Optical Pattern," Photonics Technology Letters IEEE 27(18), 1949-1952 (2015).

[39] Agrawal, G. P., [Nonlinear fiber optics] Academic Press, San Diego (2001).

[40] Chong, A., Buckley, J., Renninger, W. et al., "All-normal-dispersion femtosecond fiber laser," Opt. Express 14(21), 10095-10100 (2006).

[41] Agrawal, G. P., "Modulation instability induced by cross-phase modulation," Physical Review Letters 59(8), 880-883 (1987).

[42] Leo, F., Coen, S., Kockaert, P. et al., "Temporal cavity solitons in one-dimensional Kerr media as bits in an alloptical buffer," Nat Photonics 4(7), 471-476 (2010).

[43] Wei, X., Xu, J., Xu, Y. et al., "Breathing laser as an inertia-free swept source for high-quality ultrafast optical bioimaging," Opt. Lett. 39(23), 6593-6596 (2014).

[44] Kobtsev, S. M., and Smirnov, S. V., "Coherent properties of super-continuum containing clearly defined solitons," Opt. Express 14(9), 3968-3980 (2006).

[45] Bellini, M., and Hänsch, T. W., "Phase-locked white-light continuum pulses: toward a universal optical frequency-comb synthesizer," Opt. Lett. 25(14), 1049-1051 (2000).

[46] Myslivets, E., Alic, N., Moro, S. et al., "1.56- $\mu$ s continuously tunable parametric delay line for a 40-Gb/s signal," Opt. Express 17(14), 11958-11964 (2009).

[47] Wabnitz, S., "Optical turbulence in fiber lasers," Opt. Lett. 39(6), 1362-1365 (2014).

[48] Masoller, C., "Spatiotemporal dynamics in the coherence collapsed regime of semiconductor lasers with optical feedback," Chaos 7(3), 455-462 (1997).

[49] Kärtner, F. X., Zumbühl, D. M., and Matuschek, N., "Turbulence in Mode-Locked Lasers," Physical Review Letters 82(22), 4428-4431 (1999).

[50] Dudley, J. M., Dias, F., Erkintalo, M. et al., "Instabilities, breathers and rogue waves in optics," Nat Photon 8(10), 755-764 (2014).

[51] Solli, D. R., Ropers, C., and Jalali, B., "Rare frustration of optical supercontinuum generation," Applied Physics Letters 96(15), 151108 (2010). 